\title{
Working to prevent anal cancer through innovation
}

\author{
Stephen E. Goldstone ${ }^{1}$
}

Published online: 7 May 2021

(c) Springer Nature Switzerland AG 2021

Anal high-grade squamous intraepithelial lesions (HSIL) are the anal cancer precursor lesion and many clinicians believe that diagnosis and treatment of these lesions will prevent progression to cancer [1]. Building on the initial safety and tolerability work of Smulian et al., we first published on radiofrequency ablation (RFA) of anal HSIL in 2016 [2, 3]. In the initial study, we explored hemi-circumferential RFA as a pilot in patients who had disease limited to only $50 \%$ of the anal canal [2]. In 2017, we moved forward with circumferential ablation of the anal canal [4]. Up until this point, most HSIL ablation relied on targeted, electrocautery destruction of individual lesions. While targeted ablation was $74 \%-84 \%$ effective (depending on whether or not people were living with human immunodeficiency virus (HIV) (people living with HIV[PLWH]) at destroying individual lesions, recurrence was high because new HSIL developed over time in untreated areas (metachronous recurrence) [1]. We believed that circumferential ablation of the anal canal squamous columnar transformation zone might not only destroy HISL that had been identified but any occult HSIL as well. In fact, in our first study of hemi-circumferential RFA, we noted a diminished metachronous recurrence in the treated hemi-circumference (33\%) when compared to the treated half (14\%) [3]. We hoped that in performing circumferential RFA, we could markedly reduce overall recurrence when compared with targeted ablation as had been shown in the esophagus as a safe and effective treatment for Barrett's esophagus [5]. While the study was extremely small, we were encouraged in that all participants $(n=10)$ were HSIL-free at study completion, 12 months after their first treatment, whereas the predicted probability of recurrence with targeted ablation was greater at 53\% in PLWH $[1,4]$.

In both the hemi- circumferential and circumferential RFA trials, we only administered 3 non-overlapping RFA

Stephen E. Goldstone

Goldstone.stephen@gmail.com

1 Icahn School of Medicine At Mount Sinai New York, 420 West 23rd St, New York, NY 10011, USA pulses of $12 \mathrm{j} / \mathrm{cm}^{2} /$ application in an effort to balance an acceptable tolerability and safety profile with efficacy [3, 4]. We worried that increased energy delivery might improve efficacy but might also increase morbidity with the potential for increased pain, scarring and non-healing. At study completion we noted a high persistence rate of lesions within the first 6 months after hemi-circumferential and circumferential RFA ( $29 \%$ and $40 \%$, respectively) which we hypothesized was related to incomplete depth of destruction because of limited energy at the first treatment [3, 4]. Once lesions were retreated participants generally remained disease-free until 12 months.

As with any surgical procedure, innovative surgeons build upon the foundation laid by others to improve outcomes. As such, I was thrilled to read the results of Vergara-Fernandez et al.'s. retrospective study of circumferential RFA in PLWH [6]. While theirs was also a small trial $(n=12)$ with only 12 months of follow-up after initial RFA, they worked to decrease recurrence without sacrificing tolerability. Whereas we had only applied 3 non-overlapping pulses circumferentially to the squamocolumnar junction, Vergara-Fernandez et al. were innovative in that they set out to decrease persistence of primary lesions while still trying to prevent new lesions by applying 4 pulses of $12 \mathrm{j} / \mathrm{cm}^{2} /$ application to increase depth of destruction at sites of identifiable HSIL and only 2 pulses at sites with no apparent $\operatorname{HSIL}[3,4,6]$. The results show that at 12 months after initial treatment $58 \%$ of participants had a complete response while only $17 \%$ had persistence of the same lesion and $25 \%$ had recurrence separate from the primary HSIL [6]. When comparing the results between the 2 small trials of circumferential RFA ablation, Vergara-Fernandez et al. might have improved the technique by delivering more energy to identifiable lesions while not sacrificing efficacy and potentially diminishing morbidity by delivering less energy to apparently normal mucosa $[4,6]$.

But at what cost is efficacy potentially improved? The median pain score was $7 / 10$ the first day post-procedure falling to $4 / 10$ at 1 week. Patients did not resume normal activity until a median of 6 days post treatment [6]. The 
study is also unique in that patients took the 36-item Short Form survey of sexual function and had endoanal ultrasound and anorectal manometry prior to the first RFA and at 12 months. There were no changes in manometry or ultrasound from baseline to 12 months with unexplained improvement in sexual function after RFA. It appears that these secondary endpoints point to a safe procedure with increased pain and longer time to resume normal activities than targeted ablation.

It would be foolhardy to say that the method of 4 pulses to identifiable HSILs and 2 pulses to the rest of the canal used by Vergara-Fernandez et al. is appreciably more efficacious than the method of 3 pulses, with a similar risk profile $[3,4$, 6]. Both trials are too small to make any valid comparisons. What I can say is that the current study presents an innovative modification of circumferential RFA technique which appears safe [6]. Overall, there is more morbidity associated with circumferential ablation over targeted ablation, but there could be less recurrence. The problem is that all studies to date are single-center with very limited numbers of participants $[4,6]$. Additionally, it is fine to say that patients are disease-free at 1 year, but what about after 3 years? Do we still see a benefit of circumferential ablation over targeted treatment? Is the response durable?

\section{Declarations}

Conflict of interest Dr. Goldstone received research grant support from Medtronic Inc.
Ethical approval This article does not contain any studies with human participants or animals performed by any of the authors.

Informed consent For this type of study formal consent is not required.

\section{References}

1. Goldstone SE, Johnstone AA, Moshier EL (2014) Long-term outcome of ablation of anal high-grade squamous intraepithelial lesions: recurrence and incidence of cancer. Dis Colon Rectum 57(3):316-323

2. Smulian AG, Moore DM, Robertson JC, Kralovic SM (2014) Phase 1 study demonstrates safety and tolerability of radiofrequency ablation (RFA) of the anal mucosa. HIV Clin Trials $15: 36-44$

3. Goldstone RN, Hasan SR, Drury S, Darragh TM, van Zante A, Goldstone SE (2017) A trial of radiofrequency ablation for anal intraepithelial neoplasia. Int J Colorectal Dis 32:357-353

4. Goldstone RN, Hasan SR, Goldstone SE (2017) Brief report: radiofrequency ablation therapy for anal intraepithelial neoplasia: results from a single-center prospective pilot study in HIV + participants. J Acquir Immune Defic Syndr 76:93-97

5. Lyday WD, Corbett FS, Kuperman DA et al (2010) Radiofrequency ablation of Barrett's esophagus: outcomes of 429 patients from a multicenter community practice registry. Endoscopy 42:272-327

6. Vergara-Fernandez O, Solórzano-Vicuña D, Coss-Adame E, TrejoAvila M (2021) Outcomes of radiofrequency ablation for anal high-grade squamous intraepithelial lesions. Tech Coloproctol. https://doi.org/10.1007/s10151-020-02379-3

Publisher's Note Springer Nature remains neutral with regard to jurisdictional claims in published maps and institutional affiliations. 